\title{
A Crosslayer Geographic Routing Algorithm for the Airborne Internet
}

\author{
Daniel Medina ${ }^{\dagger}$, Felix Hoffmann ${ }^{\dagger}$, Francesco Rossetto ${ }^{\dagger}$, Carl-Herbert Rokitansky ${ }^{\ddagger}$ \\ $\dagger$ Institute of Communications and Navigation, German Aerospace Center (DLR), Munich, Germany \\ Email: $\{$ Daniel.Medina $\},\{$ Felix.Hoffmann $\},\{$ Francesco.Rossetto $\} @$ dlr.de \\ $\ddagger$ Department of Computer Science, University of Salzburg, Austria \\ Email: roki@cosy.sbg.ac.at
}

\begin{abstract}
The Airborne Internet is envisioned to be a large scale multihop wireless mesh network of commercial passenger aircraft connected via long range highly directional air-to-air radio links. We propose a localized geographic load sharing technique to mitigate congestion in this network, taking into account the underlying link scheduling constraints with directional antennas. When forwarding packets for a given destination, a node considers not one but a set of next hop candidates, and spreads traffic among them based on queue dynamics. Our simulations show that introducing this flexibility in the routing function can greatly increase a node's ability to satisfy its bandwidth demands during link scheduling, yielding significant performance improvements in terms of network throughput and average packet delay.
\end{abstract}

\section{INTRODUCTION}

Airborne mesh networks are self-organizing infrastructureless wireless networks formed by aircraft via direct air-to-air (A2A) radio links. Such networks have so far been considered mainly in the context of military aviation [1]. This paper considers the application of airborne mesh networking to commercial passenger aircraft in what has recently been termed the "Airborne Internet" [2][3]. This technology can be used to extend the coverage of broadband air-to-ground (A2G) infrastructure networks, e.g., to provide onboard Internet access in regions where aircraft are unable to reach such infrastructure, such as over large bodies of water (Fig. 1). Nowadays, connectivity in such regions is only possible via satellite links, which are costly and suffer from long round trip times. With the Airborne Internet, airlines could benefit from lower communication costs, as well as reduced round trip times, enabling delay-sensitive applications, e.g., voice over IP (VoIP).

Given the huge scale of the Airborne Internet compared to traditional mesh networks, the radio communications range must be large enough to guarantee network connectivity in regions and at times with low air traffic density. Line of sight between two aircraft is limited by the earth's curvature and depends on the aircraft's flight altitude. At $35000 \mathrm{ft}$, line-of-sight communication could in principle be achieved between two aircraft as far as $400 \mathrm{nmi}$ (nautical miles) away. At such distances, the use of highly directional antennas is crucial in order to achieve broadband communication. Directional transmission may be realized using different technologies, such as adaptive arrays or even optical

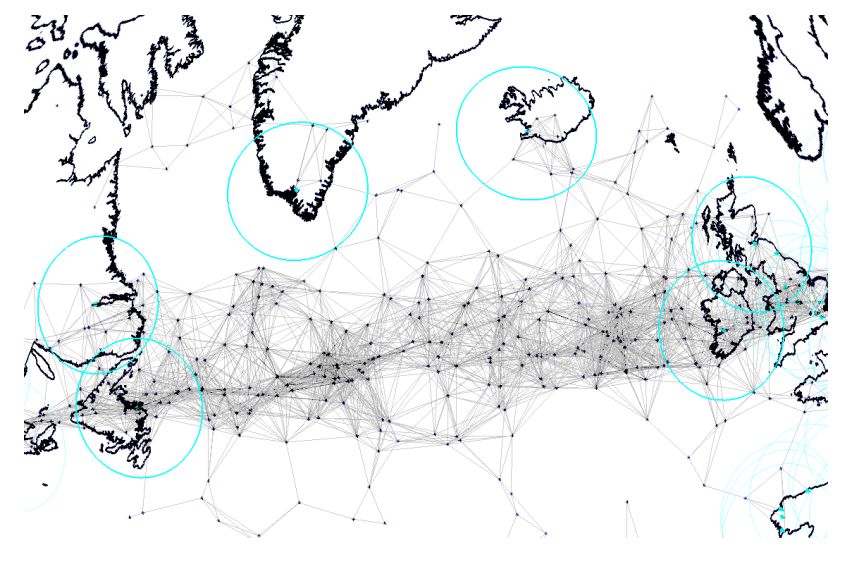

Fig. 1. The Airborne Internet.

communication links. The main disadvantage of optical links is their limited applicability in the presence of weather, such as clouds, rain, etc. Aircraft typically fly above the clouds, but the line of sight between two aircraft will typically traverse a portion of the troposphere if long range communication is to be realized.

Another key advantage of directional antennas is increased spatial reuse. By directing the beam toward the intended receiver, the energy radiated in other directions is minimal, and therefore the number of interfered nodes is reduced. If beamforming is also used in reception, the increase in spatial reuse is even higher, since the receiver rejects energy arriving from directions away from the intended transmitter. These techniques have been shown to tremendously increase the capacity of wireless networks [4][5].

Every node in the Airborne Internet is potentially hosting several hundred bandwidth-demanding users (passengers) and the aircraft may be a few hops away from the nearest Internet Gateway. Broadband air-to-air communication requires a medium access control (MAC) protocol capable of handling high traffic loads in the network and providing quality-of-service (QoS) guarantees to communicating nodes. Carrier sense multiple access (CSMA) techniques are inappropriate in this environment, since their performance degrades significantly under high traffic loads due to increased probability of collision. Aircraft are equipped with GPS for navigation purposes, and this provides a global time reference 
that can be exploited for channel access synchronization among network nodes, e.g., to schedule collision-free transmissions in a time division multiple access (TDMA) fashion.

In addition, the location information provided by GPS can be exploited for position-based forwarding of packets from source node to destination node. So-called geographic routing algorithms have been shown to scale well in large networks thanks to their localized nature [6]. Every packet contains the position of its final destination, so that intermediate nodes can forward it based on their neighbors' positions relative to the destination. This only requires a local position information exchange among neighbors, and consequently has minimal overhead.

Routing plays an important role in avoiding congestion in multihop wireless networks, and therefore has a major impact on network throughput and packet delay. In dense networks, there are typically many possible paths from source to destination, and so-called multipath routing algorithms can be used to exploit path multiplicity and improve network performance, e.g., by making use of load sharing techniques. In this paper, we propose a purely localized (and therefore scalable) geographic load share routing (GLSR) algorithm that exploits path diversity to mitigate congestion in TDMA multihop wireless networks with directional antennas.

The remainder of this paper is structured as follows. Section II provides references to some related work. Section III describes the underlying data link model used in our simulations, including the antenna and interference model and the link scheduling algorithm. Our geographic load share routing algorithm is given in Section IV. Simulation results are presented and discussed in Section V. Finally, Section VI concludes the paper.

\section{RELATED WORK}

Some attention has recently been drawn to the application of multihop wireless networking to the aeronautical environment [7]-[10]. The AeroSat Corporation, founder of the Airborne Internet Consortium (AIC) [2], has performed flight trials with directional $\mathrm{Ku}$-band antennas, targeting link data rates of up to $45 \mathrm{Mbps}$ over up to 300 nautical miles [3]. Medina et al. [11][12] recently conducted simulations of realistic air traffic to study the feasibility and characterize the network topology of such networks.

For an excellent survey on geographic routing, see [6]. An enhanced geographic routing algorithm for TDMAbased ad hoc networks is proposed by Caizzone et al. in [13]. Combined routing and scheduling with directional antennas is investigated in [14]. Jawhar et al. [15] propose an on-demand routing protocol for resource scheduling with directional antennas. In addition, our algorithm is closely related to multipath geographic routing (e.g., Popa et al. [16]). To the best of our knowledge, no work exists in the literature that addresses geographic routing in a TDMA context with directional antennas.

\section{NETWORK MODEL}

The multihop wireless network is composed of $N$ identical nodes utilizing half-duplex transceivers on the same carrier frequency (common channel). A particular node in the network is uniquely identified by its number $i, i \in\{1, \ldots, N\}$. Direct communication from node $i$ to node $j$ is represented by the directed link $(i, j), i \neq j$. A link $(i, j)$ exists if a sufficiently low bit error rate (BER) can be achieved in the absence of multiple access interference. All nodes are assumed to be synchronized to a common time reference, e.g., GPS. Interference is avoided by scheduling channel access in a TDMA fashion. Time is divided into repeating frames of size $T$ time slots. Transmissions start and end within slots. A TDMA schedule describes a node's transmission rights for each time slot in the frame.

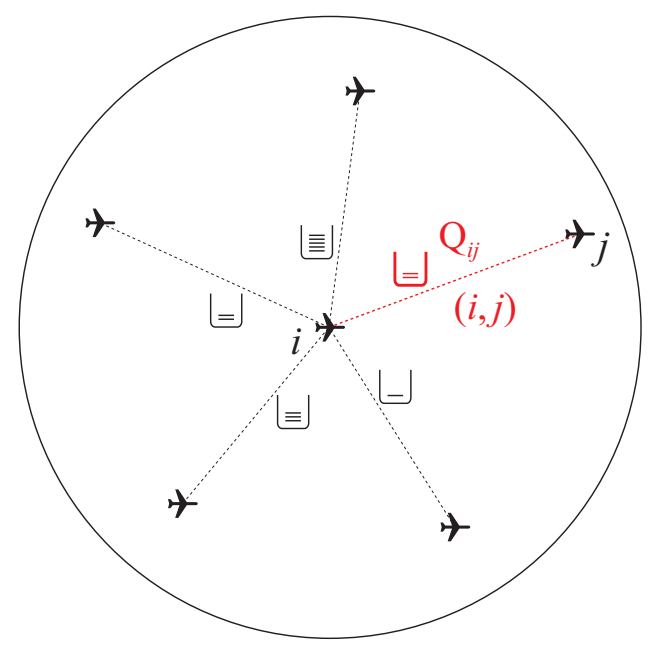

Fig. 2. A node's transmission queues.

We denote by $\mathcal{N}_{i}$ the set of all neighbors of node $i$, i.e., those nodes that are within one hop of node $i$. As shown in Fig. 2, every node $i$ has an outgoing link $(i, j)$ with each neighbor $j \in \mathcal{N}_{i}$, with an associated transmission queue $Q_{i j}$ where arriving packets are placed for eventual transmission over link $(i, j)$. The number of packets buffered in $\mathrm{Q}_{i j}$ at any time is denoted by $\mathrm{Q}_{i j}$.size. The size of a packet corresponds to the duration of a time slot minus the appropriate guard interval.

\section{A. Antenna and Interference Model}

Every node is equipped with a smart antenna capable of simultaneously forming up to $\mathcal{K}$ beams in arbitrary directions, each with a beamwidth $\psi$. The antenna is assumed to be capable of fast beam switching, so that it can quickly reconfigure the directions in which it transmits or from which it receives at the beginning of every time slot. This can be achieved by using digital signal processing (DSP) technology.

Fig. 3 illustrates the smart antenna model used. Solid lines represent transmit beams, whereas dashed lines represent receive beams. Beam patterns are modeled as circular sectors, as in [4]. We are aware that this is an 


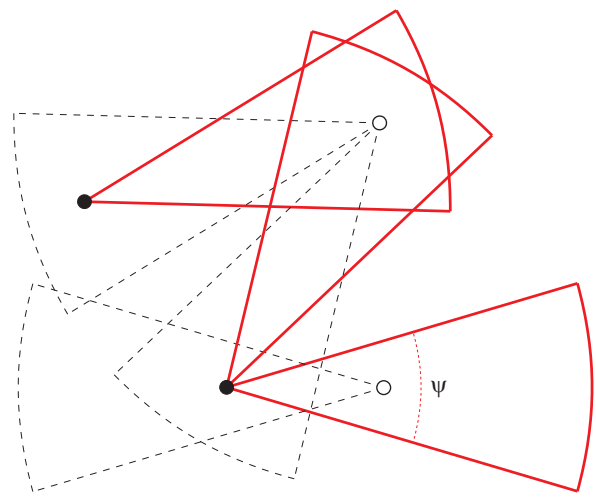

Fig. 3. Multibeam smart antenna model.

overly simplistic model, but we believe a more realistic antenna pattern and/or interference model would not change the qualitative nature of our results. Moreover, aircraft are likely to be equipped with advanced smart antenna technology capable of null steering, thus reducing the impact of side lobes.

Simultaneous link activation in a time slot is limited by the following constraints:

$\left(\mathbf{c}_{1}\right)$ Half duplex operation: A node may only either transmit or receive in a time slot, but not both.

$\left(\mathbf{c}_{2}\right)$ A node may activate at most $\mathcal{K}$ outgoing (transmit mode) or incoming (receive mode) links in a time slot.

$\left(\mathbf{c}_{3}\right)$ Hidden terminal problem: Two links $(i, j)$ and $(k, l)$ may be activated in the same time slot only if neither receiver hears both transmissions.

Fig. 4 illustrates the hidden terminal problem with directional antennas. Link $(i, j)$ is interfered by node $k^{\prime}$ s transmission to node $l$. This occurs because node $j$ is within node $k^{\prime}$ s transmit beam and node $k$ is within node $j$ 's receive beam.

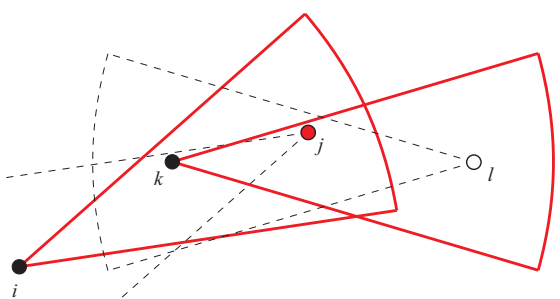

Fig. 4. Hidden terminal problem.

A special case of $\left(\mathbf{c}_{3}\right)$ occurs when $i=k$ or $j=l$ : When a certain node activates multiple outgoing/incoming links, the corresponding receivers/transmitters must be at least an angle $\psi / 2$ apart.

Because of the interference suppression achieved by TDMA scheduling and the use of directional antennas and due to the absence of fading in this propagation environment and the high transmission power available, the SINR for all compatible links is very high. We therefore consider neither packet errors nor retransmissions.

\section{B. Link Scheduling}

Spatial TDMA (STDMA) was first described by Nelson and Kleinrock [17]. We follow the distributed link scheduling approach described by Bibb Cain et al. [18], extended with a theft mechanism similar to that proposed by Grönkvist [19]. As in [19], we define the priority of a link $(i, j)$ as $p_{i j}=\lambda_{i j} / h_{i j}$, where $\lambda_{i j}$ is the packet arrival rate at $\mathrm{Q}_{i j}$ (in packets/frame) and $h_{i j}$ is the number of slots currently assigned to link $(i, j)$. This is used by link scheduling to provide fairness among competing links in the network.

In order to transmit packets over a link, a node must request transmission rights (slot allocations) from the appropriate neighbor. We define the local neighborhood $\mathcal{L}_{i}$ of node $i$ as the set of all incoming and outgoing links of $i$ 's neighbors, i.e.,

$$
\mathcal{L}_{i}=\left\{(u, v): u \in \mathcal{N}_{i}\right\} \cup\left\{(u, v): v \in \mathcal{N}_{i}\right\}
$$

Every node $i$ periodically beacons a HELLO message to its neighbors over an omnidirectional control channel (OCC). This HELLO message contains:

- the node's identity $i$,

- its current geographic location, and

- its incoming and outgoing links' slot allocations and their priority.

From the information received in these HELLO messages, node $i$ is kept up-to-date on its neighbors' locations and can maintain its local schedule $\mathcal{S}_{i}$, which reflects all allocations in its local neighborhood $\mathcal{L}_{i}$ and their priority, and will be used every time new resources (slots) need to be requested.

When a link process perceives a need for additional bandwidth, i.e., $\lambda_{i j}>h_{i j}$, it uses its local schedule $\mathcal{S}_{i}$ to find a slot where link $(i, j)$ can be activated simultaneously with already assigned links in its local neighborhood $\mathcal{L}_{i}$, according to constraints $\left(\mathbf{c}_{1}\right)-\left(\mathbf{c}_{3}\right)$ given above. The order in which slots are checked for availability is such that those slots that are furthest from slots already allocated to link $(i, j)$ are checked first. In this way, a link tries to spread its allocations evenly over the frame, which is advantageous in terms of packet delay [17].

If no slot is available, the link tries to find a slot where the removal of an allocation with lower priority than $p_{i j}$ in the local neighborhood makes the slot available for link $(i, j)$. If such is the case, this allocation will be stolen.

To formalize the assignment, nodes $i$ and $j$ perform a 3-way handshake, consisting of a REQ/REP/CONFIRM message sequence, over the OCC [18]:

1. The REQ message tells $j$ which slot is requested, and in case of theft, which allocation should be removed.

2. The REP message grants the allocation to node $i$ and informs all of $j$ 's neighbors of the new allocation (and removal, if applicable).

3. The CONFIRM message completes the 3-way handshake and informs all of $i$ 's neighbors of the new allocation (and removal, if applicable). 
This time slot assignment is maintained for as long as possible until either it can no longer be used reliably or it is stolen by a higher priority link in its local neighborhood. Node movement will cause topological changes and modify the interference geometry, so that allocations that were compatible at one time cease to be so at a later time. This leads to rescheduling, which may require additional control messages such as a RELEASE message. We will consider static topologies in our simulations. Exactly how the OCC is implemented is out of the scope of this paper. However, aircraft already use beaconing mechanisms such as ADS-B (Automatic Dependent Surveillance - Broadcast) to periodically broadcast their state vector, including position, speed, etc., and we suggest that such systems could be extended to include the above functionality.

\section{Geographic LoAd Share Routing (GLSR)}

Previous work on routing in aeronautical ad hoc networks has focused on exploiting geolocalization information [7]-[10]. In this section we describe our main contribution, the Geographic Load Share Routing (GLSR) algorithm. GLSR extends the well-known Greedy Perimeter Stateless Routing (GPSR) algorithm [20] to exploit the multiplicity of source-destination paths in moderate and high density multihop wireless networks. Greedy routing has inspired many extensions in the context of sensor networks, where energy consumption is an important performance factor [21], as well as in vehicular networks with carrier-sense multiple access schemes [22]. However, these extensions do not appear relevant to the Airborne Internet. We will therefore use GPSR as a benchmark in our simulations.

In the Airborne Internet, the distance between two nodes $i$ and $j$ is defined as the great circle angular distance $\delta_{i j}$, given (in radians) by

$$
\delta_{i j}=\cos ^{-1}\left(\sin \theta_{i} \sin \theta_{j}+\cos \theta_{i} \cos \theta_{j} \cos \left(\phi_{i}-\phi_{j}\right)\right)
$$

where $\left(\theta_{k}, \phi_{k}\right)$ denote the latitude and longitude of node $k$, respectively.

Consider a packet arriving at node $i$ with destination $m$, as shown in Fig. 5. A neighbor $k^{\prime}$ s advance toward $m$, denoted by $a_{k}^{m}$, is defined as the difference between $i^{\prime}$ s and $k^{\prime}$ s geographical distance to $m$, i.e.,

$$
a_{k}^{m}=\delta_{i m}-\delta_{k m} .
$$

In GPSR, packets are forwarded to the neighbor that is geographically closest to their destination, i.e., that yields the greatest advance. In a link scheduling context, such as STDMA, a node $i$ keeps arriving packets in a separate transmission queue $\mathrm{Q}_{i j}$ for each outgoing link $(i, j)$. Thus, GPSR places a packet arriving at node $i$ with destination $m$ in $\mathrm{Q}_{i j}$ such that

$$
a_{j}^{m}=\max _{k \in \mathcal{N}_{i}}\left\{a_{k}^{m}\right\}, a_{k}^{m}>0
$$

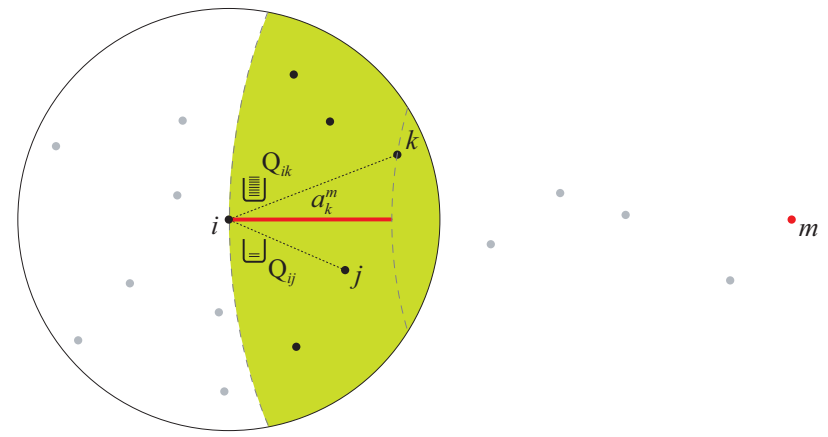

Fig. 5. Geographic Load Share Routing (GLSR).

If the packet arrival rate at $\mathrm{Q}_{i j}$ is higher than the number of slots assigned to link $(i, j)$, i.e., $\lambda_{i j}>h_{i j}$, the queue will grow in size, since packets arrive at a greater rate than they are transmitted (see Fig. 5). This will lead to increased queuing delay of packets, and may eventually result in packets being dropped, unless link $(i, j)$ is able to obtain additional slots.

In order to reduce the probability of link congestion, we introduce GLSR. We define the speed of advance $v_{k}^{m}$ of a neighbor $k$ toward destination $m$ as

$$
v_{k}^{m}=\frac{a_{k}^{m}}{\mathrm{Q}_{i k} . \text { size }+1} .
$$

GLSR places a packet arriving at node $i$ with destination $m$ in $\mathrm{Q}_{i j}$ such that

$$
v_{j}^{m}=\max _{k \in \mathcal{N}_{i}}\left\{v_{k}^{m}\right\}, v_{k}^{m}>0 .
$$

Intuitively, the idea behind GLSR is to choose the neighbor which simultaneously maximizes advance toward destination, as in GPSR, and minimizes queueing delay, as in a Join the Shortest Queue (JSQ) discipline. However, these two objectives cannot in general be fulfilled simultaneously. Thus, the solution adopted by GLSR is to maximize the ratio between advance and queueing delay, represented by the speed of advance metric. In this way, GLSR performs load sharing among all neighbors with positive advance (shaded area in Fig. 5), choosing at any time the next hop with the highest speed of advance toward the destination.

Note that information about the queue size is local to the forwarding node and does not need to be sent over the channel, thus introducing no additional overhead. If the destination $m$ is a neighbor of node $i$, the packet is simply placed in $\mathrm{Q}_{i m}$.

\section{Simulation Results}

We have conducted simulations using the OMNeT++ discrete event network simulator along with the INET framework. As shown in Fig. 6, we uniformly distribute 100 aircraft nodes over a rectangular area of size $800 \mathrm{nmi}$ x $400 \mathrm{nmi}$, according to a two dimensional Poisson point process. This area corresponds roughly to half the size of the North Atlantic Corridor (Fig. 1). The number of aircraft corresponds approximately to the 
peak instantaneous aircraft count in this region [11]. An additional ground station node is placed on one side of the rectangle, and acts as an Internet Gateway (IGW). We assume multibeam capability only at the IGW, i.e., $\mathcal{K}_{\mathrm{IGW}} \geq 1, \mathcal{K}_{\mathrm{AC}}=1$. Other than this, the IGW is just as any other node. The radio communications range, both air-to-ground and air-to-air, is set at $r=200 \mathrm{nmi}$. Note that in a real environment, as shown in Fig. 1, there may be more than one IGW available at a given time. However, there will be times where connectivity is restricted to one single IGW. Such critical situations most clearly highlight the ability of GLSR to mitigate congestion.

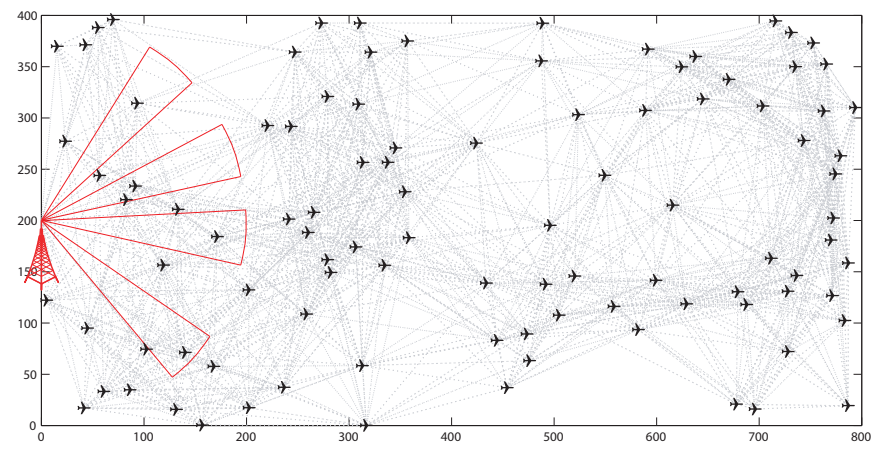

Fig. 6. Simulation scenario.

Internet traffic is generated at the IGW based on a Poisson traffic model with mean value $\lambda$ packets/slot. Each new packet has a randomly chosen destination among the 100 aircraft nodes. We do not consider upstream traffic, since most traffic in the Airborne Internet is expected to be downstream, i.e., from the Internet Gateway(s) to the aircraft. Each simulation run corresponds to a random network topology and has a length of 1000 frames, long enough to collect the metrics of interest. Queues are assumed to have infinite length.

Fig. 7 shows the worst-case per-node throughput and average end-to-end delay, averaged over 100 random topologies, with the simulation settings given in Table I. With GPSR, the fact that an intermediate node along the source-destination path cannot transmit to the next hop while receiving from the previous hop limits the maximum achievable end-to-end throughput to 0.5 packets/slot. If, in addition, this intermediate node is at a traffic crossroads in the network, i.e., is responsible for forwarding packets belonging to more than one flow, such as the IGW in our setting, the throughput will only be throttled further down. Multibeam capability alleviates this effect to some extent, since the crossroads node may be able to serve several flows simultaneously via different beams. This can be seen in Fig. 7a.

With GLSR, a node performs load sharing among all available neighbors in the direction of the destination, spatially spreading traffic based on queue dynamics. An interesting phenomenon that can be observed in these results is the extent to which GLSR exploits a node's multibeam capability. With a $\mathcal{K}$-beam antenna, the GLSR
TABLE I

SIMULATION SETTINGS

\begin{tabular}{ll}
\hline$N$ & 100 nodes \\
$r$ & $200 \mathrm{nmi}$ \\
$\psi$ & $30^{\circ}$ \\
$\mathcal{K}_{\mathrm{IGW}}$ & 1,4 beams \\
$\mathcal{K}_{\mathrm{AC}}$ & 1 beam \\
$T$ & 20 slots \\
\hline
\end{tabular}

throughput approaches $\mathcal{K} / N$ packets/slot, which is the maximum achievable throughput per node, since the IGW cannot inject into the network more than $\mathcal{K}$ packets in a slot.

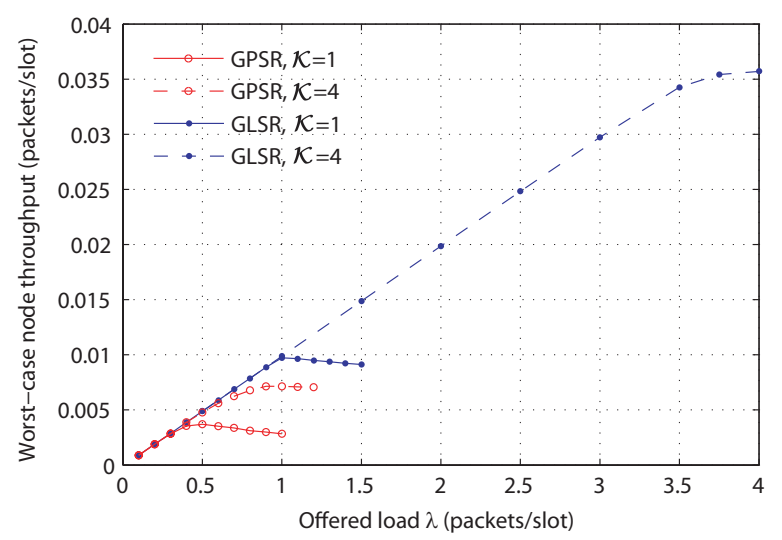

(a) Throughput

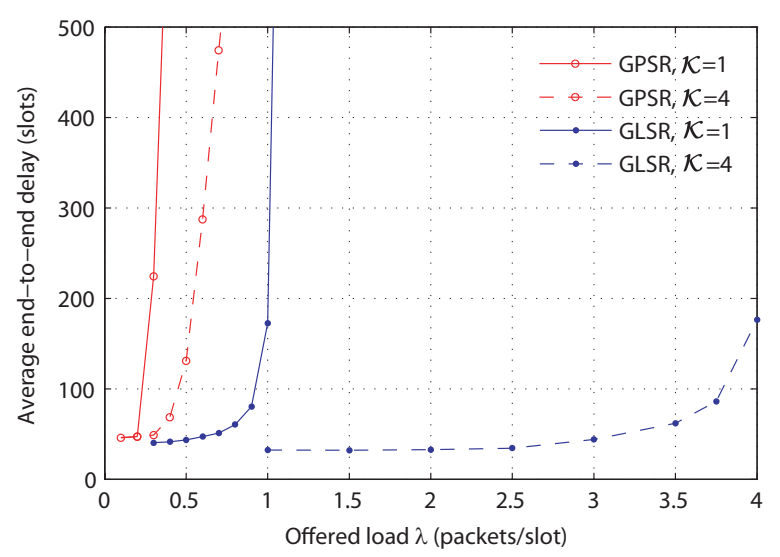

(b) Delay

Fig. 7. Simulation results for the settings given in Table I.

Fig. $7 \mathrm{~b}$ shows the average end-to-end packet delay in the network. At a certain load value in each setting, the network reaches saturation, and packets suffer increasing queueing delays at intermediate nodes. The maximum load that can be sustained with bounded delay is significantly increased by GLSR with respect to GPSR.

Fig. 8 illustrates the link activity resulting from GPSR and GLSR routing, for $\mathcal{K}_{\mathrm{IGW}}=4$ beams. By virtue of its flexibility, GLSR exploits the diversity of paths available from source to destination, dramatically increasing network throughput as compared to GPSR. 


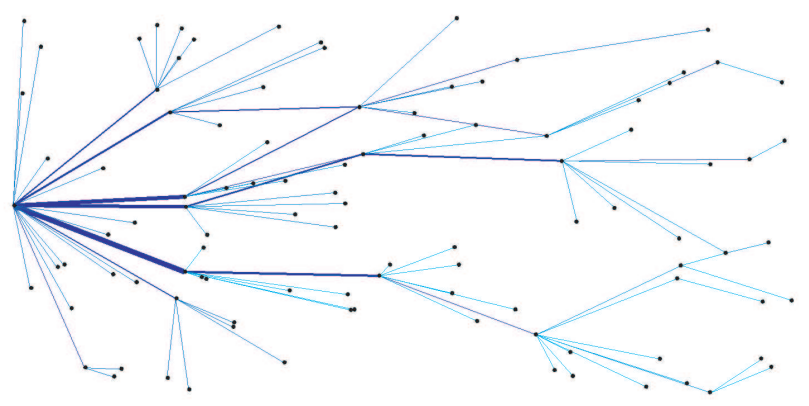

(a) GPSR

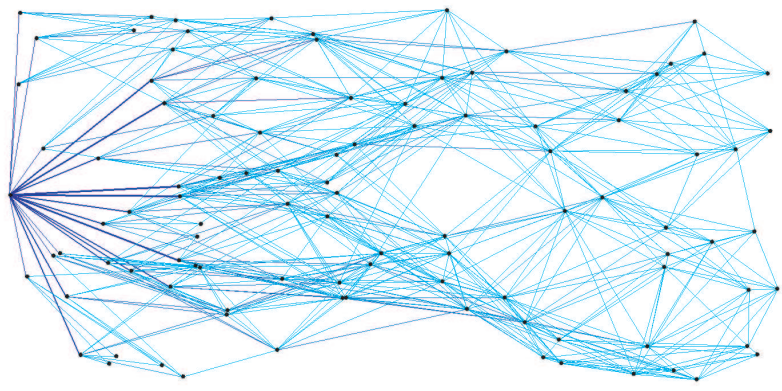

(b) GLSR

Fig. 8. Comparison of GPSR and GLSR active links. (Link width represents packet arrival rate at corresponding queue.)

\section{CONCLUSION}

The research work presented in this paper is motivated by the unique characteristics of the so-called Airborne Internet, an envisioned large scale wireless mesh network formed by commercial passenger aircraft networked with each other via highly directional air-to-air radio links. The introduction of directional antennas affords an interesting opportunity to combine position-based forwarding and channel access scheduling in multihop wireless networks. When directional antennas are used, channel access constraints are very different from those pertaining to omnidirectional antennas, and flexibility in a node's routing decision can greatly increase its ability to satisfy bandwidth demand during link scheduling.

The crosslayer principle proposed in this paper can be summarized as follows. Instead of using a single route or path through the network, locally represented by the next hop, consider a set of possible alternative paths, locally represented by a set of next hop candidates. By introducing this flexibility in the routing function, a node can perform load balancing among next hop candidates, and in so doing improve its ability to obtain transmission rights, therefore increasing the network throughput and reducing the average end-toend packet delay. Even with a single-beam antenna, our simulations show a factor-3 throughput improvement for the simulated scenario. Exactly how much can be gained depends not only on the antenna characteristics, but also on the network density and traffic matrix. Future work will focus on an analytical framework to quantify the theoretical performance gain that can be achieved with GLSR as a function of these parameters.

\section{REFERENCES}

[1] DirecNet Task Force Open Session, San Diego, February 2007.

[2] Airborne Internet Consortium (AIC), http://www.airborneinternet.org

[3] W. McNary, Transformational Aircraft Communication Using a Broadband Mesh Network, 7th ICNS Conference, May 2007.

[4] S. Yi and Y. Pei, On the Capacity Improvement of Ad Hoc Wireless Networks using Directional Antennas, ACM MobiHoc 2003, Annapolis, MD, June 2003.

[5] R. Ramanathan, J. Redi, C. Santiváñez, D. Wiggins and S. Polit, Ad Hoc Networking with Directional Antennas: A Complete System Solution, IEEE Journal on Selected Areas in Communications, March 2005, Volume 23, Issue 3, pp. 496-506.
[6] M. Mauve, J. Widmer and H. Hartenstein, A Survey on Positionbased Routing in Mobile Ad Hoc Networks, IEEE Network, November 2001, Volume 15, Issue 6, pp. 30-39.

[7] E. Sakhaee and A. Jamalipour, The Global In-Flight Internet, IEEE Journal on Selected Areas in Communications, September 2006.

[8] E. Sakhaee, A. Jamalipour and N. Kato, Aeronautical Ad Hoc Networks, IEEE WCNC 2006.

[9] M. Iordanakis, D. Yannis, K. Karras, G. Bogdos, G. Dilintas, M. Amirfeiz, G. Colangelo and S. Baiotti, Ad-hoc Routing Protocol for Aeronautical Mobile Ad-Hoc Networks, 5th Int. Symposium on Communication Systems, Networks and Digital Signal Processing (CSNDSP), July 2006.

[10] Ho Dac Tu and S. Shimamoto, Mobile Ad-Hoc Network Based Relaying Data System for Oceanic Flight Routes in Aeronautical Communications, International Journal of Computer Networks and Communications (IJCNC), Volume 1, Issue 1, April 2009.

[11] D. Medina, F. Hoffmann, S. Ayaz and C.-H. Rokitansky, Feasibility of an Aeronautical Mobile Ad Hoc Network Over the North Atlantic Corridor, IEEE SECON 2008, San Francisco, CA, June 2008.

[12] D. Medina, F. Hoffmann, S. Ayaz and C.-H. Rokitansky, Topology Characterization of High Density Airspace Aeronautical Ad Hoc Networks, IEEE MASS 2008, Atlanta, GA, September 2008.

[13] G. Caizzone, W. Erangoli, P. Giacomazzi and G. Verticale, An Enhanced GPSR Routing Algorithm for TDMA-based Ad-Hoc Networks, IEEE GLOBECOM 2005, St. Louis, MO, December 2005.

[14] M. Sánchez, Multiple Access Protocols with Smart Antennas in Multihop Ad Hoc Rural-Area Networks, Licentiate Thesis, Royal Inst. of Technology (KTH), Stockholm, 2002.

[15] I. Jawhar and J. Wu, A Routing Protocol for Resource Scheduling in Wireless Networks Using Directional Antennas, EURASIP Journal on Wireless Communications and Networking, 2008, Volume 5.

[16] L. Popa, C. Raiciu, I. Stoica and D. S. Rosenblum, Reducing Congestion Effects by Multipath Routing in Wireless Networks, 14th IEEE ICNP 2006, Santa Barbara, CA, November 2006.

[17] R. Nelson and L. Kleinrock, Spatial TDMA: A Collision-Free Multihop Channel Access Protocol, IEEE Transactions on Communications, September 1985, Volume 33, Issue 9, pp. 934-944.

[18] J. Bibb Cain, T. Billhartz, L. Foore, E. Althouse and J. Schlorff, A Link Scheduling and Ad hoc Networking Approach using Directional Antennas, IEEE MILCOM 2003, Volume 22, Issue 1, October 2003.

[19] J. Grönkvist, Interference-based Scheduling in Spatial Reuse TDMA PhD Thesis, Royal Inst. of Technology (KTH), Stockholm, 2005.

[20] B. Karp and H. T. Kung, Greedy Perimeter Stateless Routing for Wireless Networks, in Proceedings of the Sixth Annual ACM/IEEE International Conference on Mobile Computing and Networking (MobiCom 2000), Boston, MA, pp. 243-254, August 2000.

[21] T. Melodia, D. Pompili and I. F. Akyildiz, On the Interdependence of Distributed Topolooy Control and Geographical Routing in Ad Hoc and Sensor Networks, IEEE Journal of Selected Areas in Communications (JSAC), Volume 23, Issue 3, pp. 520-532, March 2005.

[22] A. Festag, R. Baldessari and H. Wang, On Power-Aware Greedy Forwarding in Highway Scenarios, Proceedings of 5th International Workshop on Intelligent Transportation (WIT07), March 2007.

[23] D. Chen and P. Varshney, A Survey of Void Handling Techniques for Geographic Routing in Wireless Networks, IEEE Communications Surveys and Tutorials, pp. 50-67, 2007. 